\title{
Stability and convergence analysis of hybrid algorithms for Berinde contraction mappings and its applications
}

\author{
Raweerote Suparatulatorn ${ }^{\mathrm{a}}$, Suthep Suantai ${ }^{\mathrm{a}}$ \\ ${ }^{a}$ Department of Mathematics, Faculty of Science, Chiang Mai University, Chiang Mai, Thailand.
}

\begin{abstract}
In this paper, we construct a new hybrid iteration, called SR-iteration, and prove its stability and convergence analysis for weak contraction mappings in a Banach space. We compare the rate of convergence between the SR-iteration and other iterations. Moreover, we provide numerical comparisons for supporting our main theorem and apply our main result to prove the existence problem of mixed type Volterra-Fredholm functional nonlinear integral equation.
\end{abstract}

Keywords: SR-iteration, rate of convergence, weak contraction, Banach space.

2020 MSC: 41A25, 47H09, 47H10.

\section{Introduction and preliminaries}

Let $C$ be a nonempty convex subset of a Banach space $X$, and $T: C \rightarrow C$ be a mapping. The fixed point set of $T$ is denoted by $F(T)$, that is, $F(T)=\{x \in C: x=T x\}$. Fixed point theory plays very important role in solving various nonlinear equations. Many iteration methods were introduced extensively by a huge number of mathematicians for approximating solutions of the studied problems, see [1, 2, 3]. For a class of contraction mappings, Picard iteration is a powerful and efficient method for approximating a fixed point of a contraction mapping. Renowned Picard iteration [4] is formulated as follows: $x_{1} \in C$ and

$$
x_{n+1}=T x_{n},
$$

for all $n \in \mathbb{N}$.

Email addresses: raweerote.s@gmail.com (Raweerote Suparatulatorn), suthep.s@cmu.ac.th (Suthep Suantai) 
In 1953, Mann [5] introduced an iteration as follows: $x_{1} \in C$ and

$$
x_{n+1}=\left(1-\alpha_{n}\right) x_{n}+\alpha_{n} T x_{n},
$$

for all $n \in \mathbb{N}$, where $\left\{\alpha_{n}\right\}$ is a sequence in $[0,1]$.

In 1974, Ishikawa [6] introduced an iteration as follows: $x_{1} \in C$ and

$$
\begin{aligned}
y_{n} & =\left(1-\beta_{n}\right) x_{n}+\beta_{n} T x_{n}, \\
x_{n+1} & =\left(1-\alpha_{n}\right) x_{n}+\alpha_{n} T y_{n},
\end{aligned}
$$

for all $n \in \mathbb{N}$, where $\left\{\alpha_{n}\right\}$ and $\left\{\beta_{n}\right\}$ are sequences in $[0,1]$.

In 2000, Noor [7] introduced a there-step iteration as follows: $h_{1} \in C$ and

$$
\begin{aligned}
l_{n} & =\left(1-\gamma_{n}\right) h_{n}+\gamma_{n} T h_{n}, \\
k_{n} & =\left(1-\beta_{n}\right) h_{n}+\beta_{n} T l_{n}, \\
h_{n+1} & =\left(1-\alpha_{n}\right) h_{n}+\alpha_{n} T k_{n},
\end{aligned}
$$

for all $n \in \mathbb{N}$, where $\left\{\alpha_{n}\right\},\left\{\beta_{n}\right\}$, and $\left\{\gamma_{n}\right\}$ are sequences in $[0,1]$.

In 2011, Phuengrattana and Suantai [8] introduced an iteration, called SP-iteration, and studied comparison of the rate of convergence between this method and Mann, Ishikawa, Noor iterations. After that they [9] also introduced a new iteration as follows: $w_{1} \in C$ and

$$
\begin{aligned}
u_{n} & =\left(1-\gamma_{n}\right) w_{n}+\gamma_{n} T w_{n}, \\
v_{n} & =\left(1-\beta_{n}\right) u_{n}+\beta_{n} T u_{n}, \\
w_{n+1} & =\left(1-\alpha_{n}-\lambda_{n}\right) v_{n}+\alpha_{n} T v_{n}+\lambda_{n} T u_{n}
\end{aligned}
$$

for all $n \in \mathbb{N}$, where $\left\{\alpha_{n}\right\},\left\{\beta_{n}\right\},\left\{\gamma_{n}\right\},\left\{\lambda_{n}\right\}$, and $\left\{\alpha_{n}+\lambda_{n}\right\}$ are sequences in $[0,1]$. We will call it here Phuengrattana iteration.

Remark 1.1. The Phuengrattana iteration reduces to the SP-iteration when we take $\lambda_{n}=0$ for all $n \in \mathbb{N}$.

A mapping $T: C \rightarrow C$ is said to satisfy condition $(*)$ if there exist $\theta \in(0,1)$ and some $L_{1} \geq 0$ such that

$$
\|T x-T y\| \leq \theta\|x-y\|+L_{1}\|x-T x\|,
$$

for each $x, y \in C$.

Remark 1.2. Note that, by the symmetry of the distance, (3) is satisfied for all $x, y \in C$ if and only if

$$
\|T x-T y\| \leq \theta\|x-y\|+L_{1}\|y-T y\| .
$$

also holds, for all $x, y \in C$.

In 1995, Osilike [10] proved several stability results of some iteration methods for a class of mappings satisfying the condition $(*)$. Those results are generalizations and extensions of those of Rhoades [11].

In order to study the order of convergence of a real sequence $\left\{a_{n}\right\}$ converging to $a$, we usually use the well-known terminology in numerical analysis, see [12, for example.

Definition 1.3. [12] Suppose $\left\{a_{n}\right\}$ is a sequence that converges to a, with $a_{n} \neq a$ for all $n$. If positive constants $\lambda$ and $\alpha$ exist with

$$
\lim _{n \rightarrow \infty} \frac{\left|a_{n+1}-a\right|}{\left|a_{n}-a\right|^{\alpha}}=\lambda
$$

then $\left\{a_{n}\right\}$ converges to a of order $\alpha$, with asymptotic error constant $\lambda$. If $\alpha=1$ (and $\lambda<1$ ), the sequence is linearly convergent and if $\alpha=2$, the sequence is quadratically convergent. 
In 2002, Berinde [13] employed above concept for comparing the rate of convergence between the two iterative methods as follows:

Definition 1.4. [13] Let $\left\{a_{n}\right\}$ and $\left\{b_{n}\right\}$ be two sequences of positive numbers that converge to a, $b$, respectively. Assume there exists

$$
\lim _{n \rightarrow \infty} \frac{\left|a_{n}-a\right|}{\left|b_{n}-b\right|}=l
$$

(i) If $l=0$, then it is said that the sequence $\left\{a_{n}\right\}$ converges to a faster than the sequence $\left\{b_{n}\right\}$ to $b$.

(ii) If $0<l<\infty$, then we say that the sequence $\left\{a_{n}\right\}$ and $\left\{b_{n}\right\}$ have the same rate of convergence.

Definition 1.5. [13, 9] Let $C$ be a nonempty closed convex subset of a Banach space $X$ and $T: C \rightarrow C$ be a mapping. Suppose $\left\{x_{n}\right\}$ and $\left\{w_{n}\right\}$ are two iterations which converge to a fixed point $p$ of $T$. We say that $\left\{x_{n}\right\}$ converges faster than $\left\{w_{n}\right\}$ to $p$ if

$$
\lim _{n \rightarrow \infty} \frac{\left\|x_{n}-p\right\|}{\left\|w_{n}-p\right\|}=0
$$

He also introduced a new class of operators which is more general than that of Zamfirescu operators.

Definition 1.6. [13] Let $C$ be a nonempty closed convex subset of a Banach space $X$. A mapping $T: C \rightarrow C$ is said to be weak contraction if there exist a constant $\delta \in(0,1)$ and some $L \geq 0$ such that

$$
\|T x-T y\| \leq \delta\|x-y\|+L\|y-T x\|
$$

for all $x, y \in C$.

The following existence and uniqueness results can be found in [13].

Proposition 1.7. [13] Let $C$ be a nonempty closed convex subset of a Banach space $X$ and $T: C \rightarrow C$ be a weak contraction with condition $(*)$. Then $T$ has a unique fixed point. Further, the Picard iteration converges to a unique fixed point of $T$.

Remark 1.8. It is known that only weak contraction does not guarantee the uniqueness of fixed point of $T$. But if $T$ also satisfies the condition $(*)$, its fixed point must be unique.

In 2013, Phuengrattana and Suantai [9] proved the strong convergence of the Phuengrattana iteration to a fixed point of a weak contraction, and this iteration converges faster than Mann, Ishikawa and Noor iterations.

Recently, Gürsoy [14] used a Picard-S iteration, which was introduced by Gürsoy and Karakaya [15] in 2014, to approximate the unique solution of mixed type Volterra-Fredholm functional nonlinear integral equation. Many contributions on fixed point outcomes with different contractive conditions have recently been published, see also [17, 18, 19].

In this work, we introduce a new iteration, called the SR-iteration, as follows:

$$
\begin{aligned}
z_{n} & =\left(1-\gamma_{n}\right) x_{n}+\gamma_{n} T x_{n}, \\
y_{n} & =\left(1-\beta_{n}\right) T z_{n}+\beta_{n} T^{2} z_{n}, \\
x_{n+1} & =\left(1-\alpha_{n}\right) T y_{n}+\alpha_{n} T^{2} y_{n},
\end{aligned}
$$

for all $n \in \mathbb{N}$, where $x_{1} \in C,\left\{\alpha_{n}\right\},\left\{\beta_{n}\right\}$, and $\left\{\gamma_{n}\right\}$ are sequences in $[0,1]$. We prove strong convergence theorems of the SR-iteration for approximating fixed points of weak contractions in a Banach space, and also compare the rate of convergence of this iteration with Phuengrattana and Noor iterations. Moreover, we prove the stability result of the SR-iteration for a weak contraction and apply the SR-iteration to estimate the unique solution of mixed type Volterra-Fredholm functional nonlinear integral equation. 


\section{Convergence theorems}

In this section, we prove the following strong convergence theorems to fixed points of weak contractions in a Banach space.

Theorem 2.1. Let $C$ be a nonempty closed convex subset of a Banach space $X$ and $T: C \rightarrow C$ be a weak contraction with condition (*). Suppose that the sequence $\left\{x_{n}\right\}$ is defined by the SR-iteration, where $\left\{\alpha_{n}\right\}$, $\left\{\beta_{n}\right\}$, and $\left\{\gamma_{n}\right\}$ are sequences in $[0,1]$ which satisfy one of the following conditions:

(C1) $\sum_{n=1}^{\infty} \alpha_{n}=\infty ;(C 2) \sum_{n=1}^{\infty} \beta_{n}=\infty ;(C 3) \sum_{n=1}^{\infty} \gamma_{n}=\infty$.

Then $\left\{x_{n}\right\}$ converges strongly to a unique fixed point of $T$.

Proof. Let $p \in F(T)$. Then by Proposition 1.7, we have

$$
\begin{aligned}
\left\|x_{n+1}-p\right\| & =\left\|\left(1-\alpha_{n}\right) T y_{n}+\alpha_{n} T^{2} y_{n}-p\right\| \\
& \leq\left(1-\alpha_{n}\right)\left\|T y_{n}-p\right\|+\alpha_{n}\left\|T^{2} y_{n}-p\right\| \\
& \leq\left(1-\alpha_{n}\right) \theta\left\|y_{n}-p\right\|+\alpha_{n} \theta^{2}\left\|y_{n}-p\right\| \\
& =\left(1-\alpha_{n}(1-\theta)\right) \theta\left\|y_{n}-p\right\|, \\
\left\|y_{n}-p\right\| \leq & \left(1-\beta_{n}\right)\left\|T z_{n}-p\right\|+\beta_{n}\left\|T^{2} z_{n}-p\right\| \\
& \leq\left(1-\beta_{n}\right) \theta\left\|z_{n}-p\right\|+\beta_{n} \theta^{2}\left\|z_{n}-p\right\| \\
& =\left(1-\beta_{n}(1-\theta)\right) \theta\left\|z_{n}-p\right\|,
\end{aligned}
$$

and

$$
\begin{aligned}
\left\|z_{n}-p\right\| & \leq\left(1-\gamma_{n}\right)\left\|x_{n}-p\right\|+\gamma_{n}\left\|T x_{n}-p\right\| \\
& \leq\left(1-\gamma_{n}\right)\left\|x_{n}-p\right\|+\gamma_{n} \theta\left\|T x_{n}-p\right\| \\
& =\left(1-\gamma_{n}(1-\theta)\right)\left\|x_{n}-p\right\| .
\end{aligned}
$$

Thus,

$$
\begin{aligned}
\left\|x_{n+1}-p\right\| & \leq \theta^{2}\left(1-\alpha_{n}(1-\theta)\right)\left(1-\beta_{n}(1-\theta)\right)\left(1-\gamma_{n}(1-\theta)\right)\left\|x_{n}-p\right\| \\
& \vdots \\
& \leq \theta^{2 n} \prod_{k=1}^{n}\left(1-\alpha_{k}(1-\theta)\right)\left(1-\beta_{k}(1-\theta)\right)\left(1-\gamma_{k}(1-\theta)\right)\left\|x_{1}-p\right\| .
\end{aligned}
$$

By the assumption, we can conclude that $\left\{x_{n}\right\}$ converges to $p$.

Theorem 2.2. [9] Assume $X, C, T$ are as in Theorem 2.1. Suppose that the sequence $\left\{w_{n}\right\}$ is defined by the Phuengrattana iteration and the sequences $\left\{\alpha_{n}\right\},\left\{\beta_{n}\right\},\left\{\gamma_{n}\right\},\left\{\lambda_{n}\right\}$, and $\left\{\alpha_{n}+\lambda_{n}\right\}$ are in $[0,1]$ which satisfy one of the conditions $(C 1),(C 2),(C 3)$ in Theorem 2.1. Then $\left\{w_{n}\right\}$ converges strongly to a unique fixed point of $T$.

Theorem 2.3. [9] Assume $X, C, T$ are as in Theorem 2.1. Suppose that the sequence $\left\{h_{n}\right\}$ is defined by the Noor iteration and the sequences $\left\{\alpha_{n}\right\},\left\{\beta_{n}\right\}$ and $\left\{\gamma_{n}\right\}$ are in $[0,1]$ such that $\sum_{n=1}^{\infty} \alpha_{n}=\infty$. Then $\left\{h_{n}\right\}$ converges strongly to a unique fixed point of $T$. 


\section{Rate of convergence}

In this section, we compare the rate of convergence between the SR-iteration and Phuengrattana iteration and Noor iteration.

Theorem 3.1. Assume $X, C, T$ are as in Theorem 2.1. Suppose $\left\{x_{n}\right\},\left\{w_{n}\right\}$ and $\left\{h_{n}\right\}$ are sequences generated by SR-iteration, Phuengrattana iteration, and Noor iteration, respectively, where $x_{1}=w_{1}=h_{1} \in C$, and the sequences $\left\{\alpha_{n}\right\},\left\{\beta_{n}\right\},\left\{\gamma_{n}\right\},\left\{\lambda_{n}\right\}$, and $\left\{\alpha_{n}+\lambda_{n}\right\}$ are in $[0,1]$ and the sequences $\left\{\alpha_{n}\right\},\left\{\beta_{n}\right\},\left\{\gamma_{n}\right\}$ satisfy one of the conditions $(C 1),(C 2),(C 3)$ in Theorem 2.1. Then

(i) If $\lim _{n \rightarrow \infty}\left(\alpha_{n}+\beta_{n}+\lambda_{n}\right)=0=\lim _{n \rightarrow \infty} \gamma_{n}$,

then $\left\{x_{n}\right\}$ converges faster than $\left\{w_{n}\right\}$ to a unique fixed point of $T$.

(ii) If $\lim _{n \rightarrow \infty} \alpha_{n}=\lim _{n \rightarrow \infty} \beta_{n}=\lim _{n \rightarrow \infty} \gamma_{n}=0$ and $\sum_{n=1}^{\infty} \alpha_{n}=\infty$,

then $\left\{x_{n}\right\}$ converges faster than $\left\{h_{n}\right\}$ to a unique fixed point of $T$.

Proof. By Theorems 2.1, 2.2 and 2.3, the sequence $\left\{x_{n}\right\},\left\{w_{n}\right\}$ and $\left\{h_{n}\right\}$ converge to a unique fixed point of $T$, say $p$.

(i) Assume that $\lim _{n \rightarrow \infty} \alpha_{n}+\beta_{n}+\lambda_{n}=0=\lim _{n \rightarrow \infty} \gamma_{n}$. From Phuengrattana iteration, we have

$$
\begin{aligned}
& \left\|w_{n+1}-p\right\|=\left\|\left(1-\alpha_{n}-\lambda_{n}\right) v_{n}+\alpha_{n} T v_{n}+\lambda_{n} T u_{n}-p\right\| \\
& \geq\left(1-\alpha_{n}-\lambda_{n}\right)\left\|v_{n}-p\right\|-\alpha_{n}\left\|T v_{n}-p\right\|-\lambda_{n}\left\|T u_{n}-p\right\| \\
& \geq\left(1-\alpha_{n}-\lambda_{n}\right)\left\|v_{n}-p\right\|-\alpha_{n} \theta\left\|v_{n}-p\right\|-\lambda_{n} \theta\left\|u_{n}-p\right\| \\
& =\left(1-\alpha_{n}(1+\theta)-\lambda_{n}\right)\left\|v_{n}-p\right\|-\lambda_{n} \theta\left\|u_{n}-p\right\| \\
& \geq\left(1-\alpha_{n}(1+\theta)-\lambda_{n}\right)\left(1-\beta_{n}-\beta_{n} \theta\right)\left\|u_{n}-p\right\|-\lambda_{n} \theta\left\|u_{n}-p\right\| \\
& =\left[\left(1-\alpha_{n}(1+\theta)-\lambda_{n}\right)\left(1-\beta_{n}(1+\theta)\right)-\lambda_{n} \theta\right]\left\|u_{n}-p\right\| \\
& =\left[1-\beta_{n}(1+\theta)-\alpha_{n}(1+\theta)\left(1-\beta_{n}(1+\theta)\right)-\lambda_{n}\left(1-\beta_{n}(1+\theta)-\lambda_{n} \theta\right]\left\|u_{n}-p\right\|\right. \\
& =\left[1-\alpha_{n}(1+\theta)\left(1-\beta_{n}(1+\theta)\right)-\beta_{n}(1+\theta)\left(1-\lambda_{n}\right)-\lambda_{n}(1+\theta)\right]\left\|u_{n}-p\right\| \\
& \geq\left(1-\left(\alpha_{n}+\beta_{n}+\lambda_{n}\right)(1+\theta)\right)\left(1-\gamma_{n}(1+\theta)\right)\left\|w_{n}-p\right\| \\
& \vdots \\
& \geq \prod_{k=1}^{n}\left(1-\left(\alpha_{k}+\beta_{k}+\lambda_{k}\right)(1+\theta)\right)\left(1-\gamma_{k}(1+\theta)\right)\left\|w_{1}-p\right\| .
\end{aligned}
$$

Thus

$$
\frac{1}{\left\|w_{n+1}-p\right\|} \leq \frac{1}{\prod_{k=1}^{n}\left(1-\left(\alpha_{k}+\beta_{k}+\lambda_{k}\right)(1+\theta)\right)\left(1-\gamma_{k}(1+\theta)\right)\left\|w_{1}-p\right\|}
$$

By inequalities (5), (6) and the assumption, we have

$$
\frac{\left\|x_{n+1}-p\right\|}{\left\|w_{n+1}-p\right\|} \leq \frac{\theta^{2 n} \prod_{k=1}^{n}\left(1-\alpha_{k}(1-\theta)\right)\left(1-\beta_{k}(1-\theta)\right)\left(1-\gamma_{k}(1-\theta)\right)}{\prod_{k=1}^{n}\left(1-\left(\alpha_{k}+\beta_{k}+\lambda_{k}\right)(1+\theta)\right)\left(1-\gamma_{k}(1+\theta)\right)}
$$

Setting $\sigma_{n}=\frac{\theta^{2 n} \prod_{k=1}^{n}\left(1-\alpha_{k}(1-\theta)\right)\left(1-\beta_{k}(1-\theta)\right)\left(1-\gamma_{k}(1-\theta)\right)}{\prod_{k=1}^{n}\left(1-\left(\alpha_{k}+\beta_{k}+\lambda_{k}\right)(1+\theta)\right)\left(1-\gamma_{k}(1+\theta)\right)}$, we get

$$
\begin{aligned}
\frac{\sigma_{n+1}}{\sigma_{n}}= & \frac{\theta^{2(n+1)} \prod_{k=1}^{n+1}\left(1-\alpha_{k}(1-\theta)\right)\left(1-\beta_{k}(1-\theta)\right)\left(1-\gamma_{k}(1-\theta)\right)}{\prod_{k=1}^{n+1}\left(1-\left(\alpha_{k}+\beta_{k}+\lambda_{k}\right)(1+\theta)\right)\left(1-\gamma_{k}(1+\theta)\right)} \\
& \times \frac{\prod_{k=1}^{n}\left(1-\left(\alpha_{k}+\beta_{k}+\lambda_{k}\right)(1+\theta)\right)\left(1-\gamma_{k}(1+\theta)\right)}{\theta^{2 n} \prod_{k=1}^{n}\left(1-\alpha_{k}(1-\theta)\right)\left(1-\beta_{k}(1-\theta)\right)\left(1-\gamma_{k}(1-\theta)\right)} \\
= & \frac{\theta^{2}\left(1-\alpha_{n+1}(1-\theta)\right)\left(1-\beta_{n+1}(1-\theta)\right)\left(1-\gamma_{n+1}(1-\theta)\right)}{\left(1-\left(\alpha_{n+1}+\beta_{n+1}+\lambda_{n+1}\right)(1+\theta)\right)\left(1-\gamma_{n+1}(1+\theta)\right)},
\end{aligned}
$$


and so $\lim _{n \rightarrow \infty} \frac{\sigma_{n+1}}{\sigma_{n}}=\theta^{2}<1$. By the ratio test, it implies that $\sum_{n=1}^{\infty} \sigma_{n}<\infty$. So, $\lim _{n \rightarrow \infty} \sigma_{n}=0$, we conclude that $\left\{x_{n}\right\}$ converges faster than $\left\{w_{n}\right\}$.

(ii) Assume that $\lim _{n \rightarrow \infty} \alpha_{n}=\lim _{n \rightarrow \infty} \beta_{n}=\lim _{n \rightarrow \infty} \gamma_{n}=0$. By the proof of Theorem 2.4. in [9], we have

$$
\left\|h_{n+1}-p\right\| \geq \prod_{k=1}^{n}\left(1-\alpha_{k}(1+\theta)\right)\left\|h_{1}-p\right\| .
$$

Hence

$$
\frac{1}{\left\|h_{n+1}-p\right\|} \leq \frac{1}{\prod_{k=1}^{n}\left(1-\alpha_{k}(1+\theta)\right)\left\|h_{1}-p\right\|}
$$

It follows from (5) and (7) that

$$
\frac{\left\|x_{n+1}-p\right\|}{\left\|h_{n+1}-p\right\|} \leq \frac{\theta^{2 n} \prod_{k=1}^{n}\left(1-\alpha_{k}(1-\theta)\right)\left(1-\beta_{k}(1-\theta)\right)\left(1-\gamma_{k}(1-\theta)\right)}{\prod_{k=1}^{n}\left(1-\alpha_{k}(1+\theta)\right)} .
$$

Setting $\tau_{n}=\frac{\theta^{2 n} \prod_{k=1}^{n}\left(1-\alpha_{k}(1-\theta)\right)\left(1-\beta_{k}(1-\theta)\right)\left(1-\gamma_{k}(1-\theta)\right)}{\prod_{k=1}^{n}\left(1-\alpha_{k}(1+\theta)\right)}$, we obtain

$$
\begin{aligned}
\frac{\tau_{n+1}}{\tau_{n}}= & \frac{\theta^{2(n+1)} \prod_{k=1}^{n+1}\left(1-\alpha_{k}(1-\theta)\right)\left(1-\beta_{k}(1-\theta)\right)\left(1-\gamma_{k}(1-\theta)\right)}{\prod_{k=1}^{n+1}\left(1-\alpha_{k}(1+\theta)\right)} \\
& \times \frac{\prod_{k=1}^{n}\left(1-\alpha_{k}(1+\theta)\right)}{\theta^{2 n} \prod_{k=1}^{n}\left(1-\alpha_{k}(1-\theta)\right)\left(1-\beta_{k}(1-\theta)\right)\left(1-\gamma_{k}(1-\theta)\right)} \\
& =\frac{\theta^{2}\left(1-\alpha_{n+1}(1-\theta)\right)\left(1-\beta_{n+1}(1-\theta)\right)\left(1-\gamma_{n+1}(1-\theta)\right)}{\left(1-\alpha_{n+1}(1+\theta)\right)},
\end{aligned}
$$

and so $\lim _{n \rightarrow \infty} \frac{\tau_{n+1}}{\tau_{n}}=\theta^{2}<1$. By the ratio test, it implies that $\sum_{n=1}^{\infty} \tau_{n}<\infty$. So, $\lim _{n \rightarrow \infty} \tau_{n}=0$, we conclude that $\left\{x_{n}\right\}$ converges faster than $\left\{h_{n}\right\}$.

\section{Stability}

In this section, we prove the stability result for the SR-iteration defined by (4) for a weak contraction with condition $(*)$. We recall the concept of stability.

Definition 4.1. Let $X$ be a Banach space and $T: C \rightarrow C$ be a mapping. Suppose a point $x_{1} \in X$ and define a fixed point iteration procedure by a general relation of the form

$$
x_{n+1}=f\left(T, x_{n}\right),
$$

for all $n \in \mathbb{N}$, and $\left\{x_{n}\right\}$ converge to a fixed point $p$ of $T$. Let $\left\{y_{n}\right\}$ be an arbitrary sequence in $X$ and set

$$
\varepsilon_{n}=\left\|y_{n+1}-f\left(T, y_{n}\right)\right\| .
$$

The sequence $\left\{x_{n}\right\}$ is T-stable (or stable with respect to $T$ ) if

$$
\lim _{n \rightarrow \infty} \varepsilon_{n}=0 \text { if and only if } \lim _{n \rightarrow \infty} y_{n}=p .
$$

We now prove the stability result of the SR-iteration. 
Theorem 4.2. Let $C$ be a nonempty closed convex subset of a Banach space $X$ and $T: C \rightarrow C$ be a weak contraction with condition (*). Suppose that the sequences $\left\{x_{n}\right\}$ is defined by the SR-iteration and the sequence $\left\{\alpha_{n}\right\},\left\{\beta_{n}\right\}$, and $\left\{\gamma_{n}\right\}$ are in $[0,1]$ which satisfy one of the conditions $(C 1),(C 2),(C 3)$ in Theorem 2.1. Then the sequence $\left\{x_{n}\right\}$ is T-stable.

Proof. From Theorem 2.1, the sequence $\left\{x_{n}\right\}$ converge to a unique fixed point of $T$, say $p$. Let $\left\{p_{n}\right\}$ be an arbitrary sequence in $C$ and define

$$
\begin{aligned}
& q_{n}=\left(1-\gamma_{n}\right) p_{n}+\gamma_{n} T p_{n}, \\
& r_{n}=\left(1-\beta_{n}\right) T q_{n}+\beta_{n} T^{2} q_{n}, \\
& \varepsilon_{n}=\left\|p_{n+1}-\left(\left(1-\alpha_{n}\right) T r_{n}+\alpha_{n} T^{2} r_{n}\right)\right\|,
\end{aligned}
$$

for all $n \in \mathbb{N}$. By the same proof of Theorem 2.1, we obtain

$$
\begin{aligned}
& \left\|\left(1-\alpha_{n}\right) T r_{n}+\alpha_{n} T^{2} r_{n}-p\right\| \\
& \leq \theta^{2}\left(1-\alpha_{n}(1-\theta)\right)\left(1-\beta_{n}(1-\theta)\right)\left(1-\gamma_{n}(1-\theta)\right)\left\|p_{n}-p\right\| \\
& \vdots \\
& \leq \theta^{2 n} \prod_{k=1}^{n}\left(1-\alpha_{k}(1-\theta)\right)\left(1-\beta_{k}(1-\theta)\right)\left(1-\gamma_{k}(1-\theta)\right)\left\|p_{1}-p\right\| .
\end{aligned}
$$

Next, assume that $\lim _{n \rightarrow \infty} \varepsilon_{n}=0$. By above inequality, we have

$$
\begin{aligned}
& \left\|p_{n+1}-p\right\| \\
& \leq\left\|p_{n+1}-\left(\left(1-\alpha_{n}\right) T r_{n}+\alpha_{n} T^{2} r_{n}\right)\right\|+\left\|\left(1-\alpha_{n}\right) T r_{n}+\alpha_{n} T^{2} r_{n}-p\right\| \\
& \leq \varepsilon_{n}+\theta^{2 n} \prod_{k=1}^{n}\left(1-\alpha_{k}(1-\theta)\right)\left(1-\beta_{k}(1-\theta)\right)\left(1-\gamma_{k}(1-\theta)\right)\left\|p_{1}-p\right\| .
\end{aligned}
$$

It follows from above inequality and our assumptions on the sequences $\left\{\alpha_{n}\right\},\left\{\beta_{n}\right\}$ and $\left\{\gamma_{n}\right\}$ that $\lim _{n \rightarrow \infty} p_{n}=p$.

Conversely, assume that $\lim _{n \rightarrow \infty} p_{n}=p$, then

$$
\begin{aligned}
\varepsilon_{n} & \leq\left\|p_{n+1}-p\right\|+\left\|p-\left(\left(1-\alpha_{n}\right) T r_{n}+\alpha_{n} T^{2} r_{n}\right)\right\| \\
& \leq\left\|p_{n+1}-p\right\|+\theta^{2}\left(1-\alpha_{n}(1-\theta)\right)\left(1-\beta_{n}(1-\theta)\right)\left(1-\gamma_{n}(1-\theta)\right)\left\|p_{n}-p\right\| \\
& \leq\left\|p_{n+1}-p\right\|+\left\|p_{n}-p\right\| .
\end{aligned}
$$

By above inequality and $p_{n} \rightarrow p$ as $n \rightarrow \infty$, we obtain that $\lim _{n \rightarrow \infty} \varepsilon_{n}=0$. Therefore, the sequence $\left\{x_{n}\right\}$ is T-stable.

\section{Numerical results}

Example 5.1. Consider $\mathbb{R}^{2}$ with the Euclidean norm. Let $C=[0,1] \times[0,1]$ and $T: C \rightarrow C$ be defined as

$$
T((x, y))=\left(\sqrt{x^{2}-x+\frac{1}{2}}, \sin (\cos y)\right),
$$

for all $(x, y) \in C$. Then $T$ is a weak contraction with condition $(*)$. Suppose $\left\{x_{n}\right\},\left\{s_{n}\right\},\left\{w_{n}\right\}$, and $\left\{h_{n}\right\}$ are sequences generated by SR-iteration, SP-iteration, Phuengrattana iteration, and Noor iteration, respectively, Choose $\alpha_{n}=\frac{1}{n+1}, \beta_{n}=\frac{1}{2 n+1}, \gamma_{n}=\frac{1}{n+2}$, and $\lambda_{n}=\frac{1}{n^{2}+1}$, for all $n \in \mathbb{N}$. It is clear that sequences $\left\{\alpha_{n}\right\},\left\{\beta_{n}\right\}$, $\left\{\gamma_{n}\right\}$, and $\left\{\lambda_{n}\right\}$ satisfy all the conditions of Theorem 3.1. For the initial point $h_{1}=s_{1}=w_{1}=x_{1}=(0,0)$. We obtain the following numerical experiments for fixed point of $T$, rate of convergence and numerical experiments of the studied methods. 


\begin{tabular}{ccccc}
\hline \multirow{2}{*}{$n$} & Noor & SP & Phuengrattana & SR \\
\cline { 2 - 5 } & $h_{n}$ & $s_{n}$ & $w_{n}$ & $x_{n}$ \\
\hline 2 & $(0.29454,0.41043)$ & $(0.43447,0.62052)$ & $(0.54442,0.80026)$ & $(0.50102,0.68651)$ \\
3 & $(0.37138,0.53183)$ & $(0.47515,0.67722)$ & $(0.51320,0.70256)$ & $(0.50000,0.69441)$ \\
4 & $(0.40647,0.58606)$ & $(0.48741,0.68853)$ & $(0.50595,0.69688)$ & $(0.50000,0.69479)$ \\
$\vdots$ & $\vdots$ & $\vdots$ & $\vdots$ & $\vdots$ \\
10 & $(0.46455,0.66564)$ & $(0.49860,0.69459)$ & $(0.50055,0.69487)$ & $(0.50000,0.69482)$ \\
\hline
\end{tabular}

Table 1: Numerical experiments of Noor, SP, Phuengrattana, and SR-iterations.

\begin{tabular}{ccccc}
\hline \multirow{2}{*}{$n$} & Noor & SP & Phuengrattana & SR \\
\cline { 2 - 5 } & $\left\|h_{n}-T h_{n}\right\|$ & $\left\|s_{n}-T s_{n}\right\|$ & $\left\|w_{n}-T w_{n}\right\|$ & $\left\|x_{n}-T x_{n}\right\|$ \\
\hline 2 & 0.45548 & 0.12712 & 0.16429 & 0.01215 \\
3 & 0.26950 & 0.03610 & 0.01727 & 0.00059 \\
4 & 0.18481 & 0.01570 & 0.00664 & 0.00004 \\
$\vdots$ & $\vdots$ & $\vdots$ & $\vdots$ & $\vdots$ \\
10 & 0.05597 & 0.00145 & 0.00055 & $1.5001 \mathrm{e}-10$ \\
\hline
\end{tabular}

Table 2: Numerical experiments of the studied methods.

\begin{tabular}{ccccc}
\hline \multirow{2}{*}{$n$} & Noor & SP & Phuengrattana & SR \\
\cline { 2 - 5 } & $\left\|h_{n}-h_{n-1}\right\|$ & $\left\|s_{n}-s_{n-1}\right\|$ & $\left\|w_{n}-w_{n-1}\right\|$ & $\left\|x_{n}-x_{n-1}\right\|$ \\
\hline 2 & 0.50518 & 0.75750 & 0.96789 & 0.84989 \\
3 & 0.14368 & 0.06978 & 0.10256 & 0.00797 \\
4 & 0.06459 & 0.01669 & 0.00922 & 0.00038 \\
$\vdots$ & $\vdots$ & $\vdots$ & $\vdots$ & $\vdots$ \\
10 & 0.00631 & 0.00042 & 0.00017 & $6.0593 \mathrm{e}-10$ \\
\hline
\end{tabular}

Table 3: Numerical errors in Example 5.1

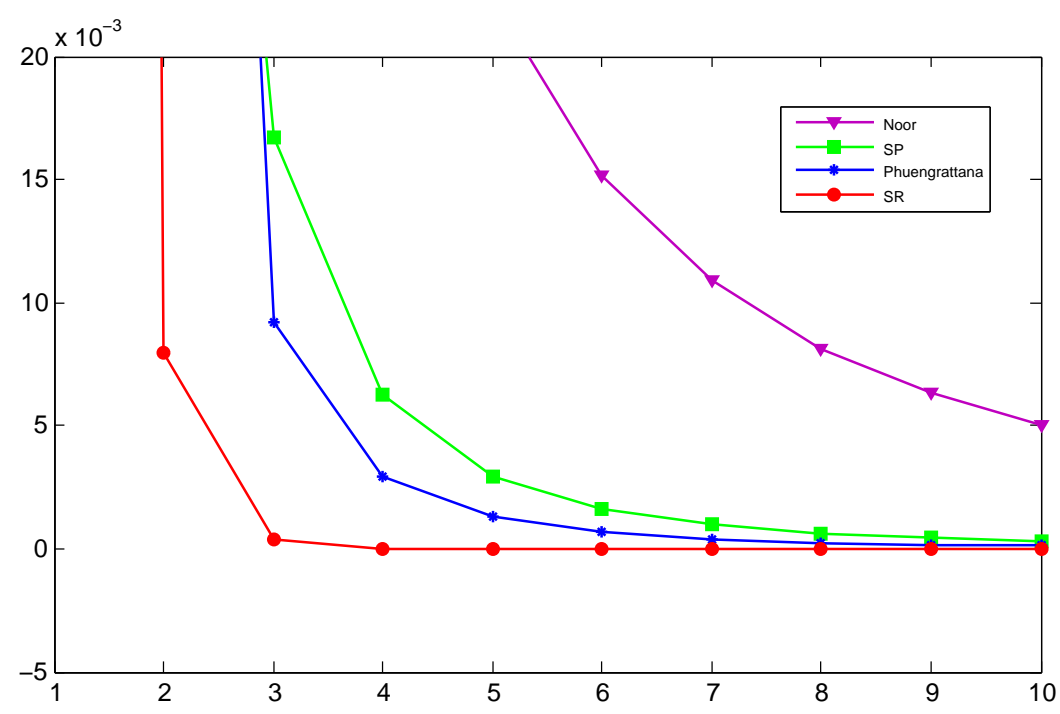

Figure 1: Comparison of errors in Example 5.1.

By Theorem 2.1, we know that the sequence $\left\{x_{n}\right\}$ converges to a unique fixed point $p$ of $T$ faster than that the others. From Tables 2 and 3 , we observe that the sequence $\left\{x_{n}\right\}$ converges faster than the others and from Tables 1 and 2 , we also note that $p \approx(0.50000,0.69482)$ with accuracy 9 D.P. 


\section{Application to mixed type Volterra-Fredholm functional nonlinear integral equation}

In this section, we use the SR-iteration to approximate the unique solution of mixed type VolterraFredholm functional nonlinear integral equation which is in the following from (see [14, 16]):

$$
x(t)=F\left(t, x(t), \int_{a_{1}}^{t_{1}} \cdots \int_{a_{m}}^{t_{m}} K(t, s, x(s)) d s, \int_{a_{1}}^{b_{1}} \cdots \int_{a_{m}}^{b_{m}} H(t, s, x(s)) d s\right),
$$

where $\left[a_{1}, b_{1}\right] \times \cdots \times\left[a_{m}, b_{m}\right]$ be an interval in $\mathbb{R}^{m}, K, H:\left[a_{1}, b_{1}\right] \times \cdots \times\left[a_{m}, b_{m}\right] \times\left[a_{1}, b_{1}\right] \times \cdots \times\left[a_{m}, b_{m}\right] \times \mathbb{R} \rightarrow \mathbb{R}$ continuous functions and $F:\left[a_{1}, b_{1}\right] \times \cdots \times\left[a_{m}, b_{m}\right] \times \mathbb{R}^{3} \rightarrow \mathbb{R}$.

Theorem 6.1. Let $X=C\left(\left[a_{1}, b_{1}\right] \times \cdots \times\left[a_{m}, b_{m}\right]\right)$ be the Banach space with the Cebyshev's norm. Assume that $T: X \rightarrow X$ is a mapping defined by

$$
T(x)(t)=F\left(t, x(t), \int_{a_{1}}^{t_{1}} \cdots \int_{a_{m}}^{t_{m}} K(t, s, x(s)) d s, \int_{a_{1}}^{b_{1}} \cdots \int_{a_{m}}^{b_{m}} H(t, s, x(s)) d s\right),
$$

for all $x \in X$. Suppose that the following condition holds:

(C4) $K, H \in C\left(\left[a_{1}, b_{1}\right] \times \cdots \times\left[a_{m}, b_{m}\right] \times\left[a_{1}, b_{1}\right] \times \cdots \times\left[a_{m}, b_{m}\right] \times \mathbb{R}\right)$;

(C5) $F \in C\left(\left[a_{1}, b_{1}\right] \times \cdots \times\left[a_{m}, b_{m}\right] \times \mathbb{R}^{3}\right)$;

(C6) there exist nonnegative constants $\kappa, \zeta, \eta$ with $\kappa<1$ such that

$$
\left|F\left(t, u_{1}, v_{1}, w_{1}\right)-F\left(t, u_{2}, v_{2}, w_{2}\right)\right| \leq \kappa\left|u_{1}-u_{2}\right|+\zeta\left|v_{1}-v_{2}\right|+\eta\left|w_{1}-w_{2}\right|,
$$

for all $t \in\left[a_{1}, b_{1}\right] \times \cdots \times\left[a_{m}, b_{m}\right], u_{i}, v_{i}, w_{i} \in \mathbb{R}, i=1,2$;

$(C 7)$ there exist nonnegative constants $L_{K}, L_{H}$ such that

$$
\begin{aligned}
& |K(t, s, x(s))-K(t, s, y(s))| \leq L_{K} \min \{|y(s)-T(x)(s)|,|y(s)-T(y)(s)|\}, \\
& |H(t, s, x(s))-H(t, s, y(s))| \leq L_{H} \min \{|y(s)-T(x)(s)|,|y(s)-T(y)(s)|\},
\end{aligned}
$$

for all $t, s \in\left[a_{1}, b_{1}\right] \times \cdots \times\left[a_{m}, b_{m}\right], x, y \in X$.

Then $T$ is a weak contraction with condition $(*)$.

Proof. Assume that $x, y \in X$ and $t, s \in\left[a_{1}, b_{1}\right] \times \cdots \times\left[a_{m}, b_{m}\right]$. Then

$$
\begin{aligned}
\| & T x-T y \|=|T(x)(t)-T(y)(t)| \\
= & \mid F\left(t, x(t), \int_{a_{1}}^{t_{1}} \cdots \int_{a_{m}}^{t_{m}} K(t, s, x(s)) d s, \int_{a_{1}}^{b_{1}} \cdots \int_{a_{m}}^{b_{m}} H(t, s, x(s)) d s\right) \\
& -F\left(t, y(t), \int_{a_{1}}^{t_{1}} \cdots \int_{a_{m}}^{t_{m}} K(t, s, y(s)) d s, \int_{a_{1}}^{b_{1}} \cdots \int_{a_{m}}^{b_{m}} H(t, s, y(s)) d s\right) \mid \\
\leq & \kappa|x(t)-y(t)|+\zeta\left|\int_{a_{1}}^{t_{1}} \cdots \int_{a_{m}}^{t_{m}} K(t, s, x(s)) d s-\int_{a_{1}}^{t_{1}} \cdots \int_{a_{m}}^{t_{m}} K(t, s, y(s)) d s\right| \\
& +\eta\left|\int_{a_{1}}^{b_{1}} \ldots \int_{a_{m}}^{b_{m}} H(t, s, x(s)) d s-\int_{a_{1}}^{b_{1}} \cdots \int_{a_{m}}^{b_{m}} H(t, s, y(s)) d s\right| \\
\leq & \kappa|x(t)-y(t)|+\zeta \int_{a_{1}}^{t_{1}} \ldots \int_{a_{m}}^{t_{m}}|K(t, s, x(s))-K(t, s, y(s))| d s \\
& +\eta \int_{a_{1}}^{b_{1}} \ldots \int_{a_{m}}^{b_{m}}|H(t, s, x(s))-H(t, s, y(s))| d s \\
\leq & \kappa|x(t)-y(t)|+\zeta \int_{a_{1}}^{t_{1}} \ldots \int_{a_{m}}^{t_{m}} L_{K} \min \{|y(s)-T(x)(s)|,|y(s)-T(y)(s)|\} d s \\
& +\eta \int_{a_{1}}^{b_{1}} \ldots \int_{a_{m}}^{b_{m}} L_{H} \min \{|y(s)-T(x)(s)|,|y(s)-T(y)(s)|\} d s \\
\leq & \kappa\|x-y\|+\left(\zeta L_{K}+\eta L_{H}\right)\left(b_{1}-a_{1}\right) \cdots\left(b_{m}-a_{m}\right) \min \{\|y-T x\|,\|y-T y\|\} .
\end{aligned}
$$

By our assumptions, we can conclude that $T$ is a weak contraction with condition $(*)$. 
The following result shows that the mixed type Volterra-Fredholm functional nonlinear integral equation (8) has a unique solution.

Theorem 6.2. Let $X=C\left(\left[a_{1}, b_{1}\right] \times \cdots \times\left[a_{m}, b_{m}\right]\right)$ be the Banach space with the Cebyshev's norm. Assume that $T: X \rightarrow X$ is a mapping defined by (9). Suppose that the conditions $(C 4)-(C 7)$ in Theorem 6.1 hold. Then the equation (8) has a unique solution, say $x^{*}$, in $X$, and the Picard iteration converges to $x^{*}$.

Proof. By Theorem 6.1, we know that $T$ is a weak contraction with condition (*). By Proposition 1.7, there exists a unique solution $x^{*}$ of the equation (8) and the Picard iteration converges to $x^{*}$.

Theorem 6.3. Let $X=C\left(\left[a_{1}, b_{1}\right] \times \cdots \times\left[a_{m}, b_{m}\right]\right)$ be the Banach space with the Cebyshev's norm. Suppose that the sequence $\left\{x_{n}\right\}$ is defined by the SR-iteration and the sequences $\left\{\alpha_{n}\right\},\left\{\beta_{n}\right\}$, and $\left\{\gamma_{n}\right\}$ are in $[0,1]$ which satisfy one of the conditions $(C 1),(C 2),(C 3)$ in Theorem 2.1. Assume that $T: X \rightarrow X$ is a mapping defined by (9). Suppose that the conditions $(C 4)-(C 7)$ in Theorem 6.1 hold. Then the SR-iteration converges to a unique solution of the equation (8).

Proof. By Theorem 2.1, Theorem 6.1, and Theorem 6.2, we can conclude that the SR-iteration converges to a unique solution of the equation (8).

Acknowledgment. This research work was partially supported by Chiang Mai University.

\section{References}

[1] W. Chaolamjiak, D. Yambangwai, H.A. Hammad, Modified hybrid projection methods with SP iterations for quasinonexpansive multivalued mappings in Hilbert spaces, Bull. Iran. Math. Soc. (2020). https://doi.org/10.1007/s41980-02000448-9.

[2] W. Cholamjiak, D. Yambangwai, H. Dutta, H.A. Hammad, Modified CQ-algorithms for G-nonexpansive mappings in Hilbert spaces involving graphs, New Math. Nat. Comput. 16(1) (2019) 89-103.

[3] W. Cholamjiak, S. Suantai, R. Suparatulatorn, S. Kesornprom, P. Cholamjiak, Viscosity approximation methods for fixed point problems in Hilbert spaces endowed with graphs, J. Appl. Numer. Optim. 1 (2019) 25-38.

[4] E. Picard, Mémoire sur la théorie des équations aux dérivées partielles et la méthode des approximations successives, J. Math. Pures Appl. 6(4) (1890) 145-210.

[5] W.R. Mann, Mean value methods in iteration, Proc. Amer. Math. Soc. 4 (1953) 506-510.

[6] S. Ishikawa, Fixed point by a new iteration method, Proc. Amer. Math. Soc. 44 (1974) 147-150.

[7] M.A. Noor, New approximation schemes for general variational inequalities, J. Math. Anal. Appl. 251(1) (2000) $217-229$.

[8] W. Phuengrattana, S. Suantai, On the rate of convergence of Mann, Ishikawa, Noor and SP-iterations for continuous functions on an arbitrary interval, J. Comput. Appl. Math. 235(9) (2011) 3006-3014.

[9] W. Phuengrattana, S. Suantai, Comparison of the rate of convergence of various iterative methods for the class of weak contractions in Banach spaces, Thai J. Math. 11(1) (2013) 217-226.

[10] M.O. Osilike, Stability results for Ishikawa fixed point iteration procedure, Indian J. Pure Appl. Math. 26(10) (1995) 937-941.

[11] B.E. Rhoades, Fixed point theorems and stability results for fixed point iteration procedures, Indian J. Pure Appl. Math. 21 (1990) 1-9.

[12] R.L. Burden, J.D. Faires, Numerical Analysis, 9th edn. Brooks/Cole Cengage Learning, Boston (2010).

[13] V. Berinde, Iterative Approximation of Fixed Points. Editura Efemeride, Baia Mare (2002).

[14] F. Gürsoy, A Picard-S iterative method for approximating fixed point of weak-contraction mappings, Filomat, 30(10) (2016) 2829-2845.

[15] F. Gürsoy, V. Karakaya, A Picard-S hybrid type iteration method for solving a differential equation with retarded argument, arXiv:1403.2546v2 (2014).

[16] C. Crăciun, M.A. Şerban, A nonlinear integral equation via Picard operators, Fixed Point Theory. 12(1) (2011) 57-70.

[17] H.A. Hammad, M. De la Sen, Solution of nonlinear integral equation via fixed point of cyclic $\alpha_{L}^{\psi}$-rational contraction mappings in metric-like spaces, Bull. Braz. Math. Soc. New Ser. 51 (2020) 81-105.

[18] H.A. Hammad, M. De la Sen, Generalized contractive mappings and related results in b-metric like spaces with an application, Symmetry, 11(5) (2019) 667.

[19] H.A. Hammad, M. De la Sen, A solution of Fredholm integral equation by using the cyclic $\eta_{s}^{q}$-rational contractive mappings technique in b-metric-like spaces, Symmetry, 11(9) (2019) 1184. 